\title{
Computation of electron precipitation atmospheric ionization: updated model CRAC-EPII
}

\author{
Alexander Mishev* \\ Space Climate Research Unit, University of Oulu, Finland. \\ E-mail: alexander.mishev@oulu.fi \\ Anton Artamonov \\ Space Climate Research Unit, University of Oulu, Finland. \\ E-mail: anton.artamonoveoulu.fi
}

Genady Kovaltsov

Ioffe Physical-Technical Institute of Russian Academy of Sciences, St. Petersburg, Russia.

E-mail: gen.koval@mail.ru

\section{Irina Mironova}

St. Petersburg State University, Institute of Physics, St. Petersburg, Russia

E-mail: irini.mironova@gmail.com

\section{Ilya Usoskin}

Space Climate Research Unit; Sodankylä Geophysical Observatory (Oulu unit), University of Oulu, Finland.

E-mail: ilya.usoskin@oulu.fi

\begin{abstract}
A new model of the CRAC family, CRAC:EPII (Cosmic Ray Atmospheric Cascade: Electron Precipitation Induced Ionization) is presented. The model allows one to calculate atmospheric ionization induced by precipitating electrons. The model is based on pre-computed with highprecision ionization yield functions, which are obtained using full Monte Carlo simulation of electron propagation and interaction in the Earth's atmosphere, explicitly considering all physical processes involved in ion production. The simulations were performed using GEANT 4 simulation tool PLANETOCOSMICS with NRLMSISE 00 atmospheric model. A quasi-analytical approach, which allows one to compute the ionization yields for events with arbitrary incidence is also presented. It is compared with Monte Carlo simulations and good agreement between Monte Carlo simulations and quasi-analytical approach is achieved.
\end{abstract}

35th International Cosmic Ray Conference - ICRC 2017-

10-20 July, 2017

Bexco, Busan, Korea

\footnotetext{
*Speaker.
} 


\section{Introduction}

Different types and populations of high energy particles are involved in the atmospheric ionization [1,2]. The energetic particles (EPs) are the main sources of ionization below $100 \mathrm{~km}$. At altitudes above $100 \mathrm{~km}$ dominate the contribution of solar UV and X-rays, which are absorbed below. Energetic precipitating particles include galactic cosmic rays (GCRs), solar energetic particles (SEPs), precipitating protons, relativistic electrons from radiation belts, auroral electrons. In this work we focus on relativistic electrons, while other sources are considered elsewhere.

The precipitating electrons ionize the atmosphere, specifically it's upper polar part. Effects due to electron precipitation are usually observed in the auroral zone [3]. However, occasionally relativistic electron precipitation can occur also in sub-auroral zone as well as in middle latitudes [4, 5]. In general, electrons precipitate into the atmosphere from different regions of the magnetosphere due to various mechanisms $[6,7,8,9,10,11]$. There are several atmospheric processes affected by the impact ionization as well as processes related to global electric circuit and minor constituents in the Earth's atmosphere $[12,13,14,15]$. In the upper part of the atmosphere the impact ionization is governed by the direct ionization, while in the lower part of the atmosphere dominates the secondary ionization mostly due to Bremsstrahlung radiation (henceforth Bremsstrahlung).

The majority of studies are focused at heights of about 60-80 km above the sea level (a.s.l.), which correspond to precipitating electrons of about 100-400 keV. However, the contribution of relativistic electron precipitation is not discussed nor the additional Bremsstrahlung ionization. Here, we present a model based on Monte Carlo simulations for computation of ionization induced by relativistic electron precipitation explicitly taking into account Bremsstrahlung.

\section{CRAC:EPII model}

In this study, the propagation and interaction of high energy protons with the atmosphere are simulated using the PLANETOCOSMICS code [16]. We employ the NRLMSISE 00 atmospheric model [17]. Here, we use a previously developed formalism of a yield function [18]. The ion production rate in the atmosphere is obtained as an integral of the product of the primary particle spectrum and the pre-computed yield function defined as:

$$
Y(x, E)=\frac{\partial E(x, E)}{E_{\text {ion }} \partial x}
$$

where $\partial E$ is the energy deposition in atmospheric layer $\partial x$ at depth $x$, averaged per primary particle with kinetic energy $E$, and $E_{i o n}=35 \mathrm{eV}$ is the average energy necessary for production of an ion pair in air [19]. The computations were carried out in the energy range of precipitating electrons between $20 \mathrm{keV}$ and $500 \mathrm{MeV}$. and at atmospheric depths from $6.5 \times 10^{-9} \mathrm{~g} / \mathrm{cm}^{2}$ (about $200 \mathrm{~km}$ a.s.1.) to the sea level $\left(1033 \mathrm{~g} / \mathrm{cm}^{2}\right)$. An example of ionization yields for electrons is shown in Fig.1 (isotropic incidence) and Fig.2 (various incidence). The ionization yield function $Y(x, E)$ convoluted with a primary particle spectrum gives the ion production rate $\mathrm{q}(\mathrm{x})$ at a given depth $x$ as

$$
q(h)=\int J(K) Y(h, K) \rho(h) d K
$$

where $\mathrm{J}(\mathrm{K})$ is the differential energy spectrum of the primary particles with energy $K$. 


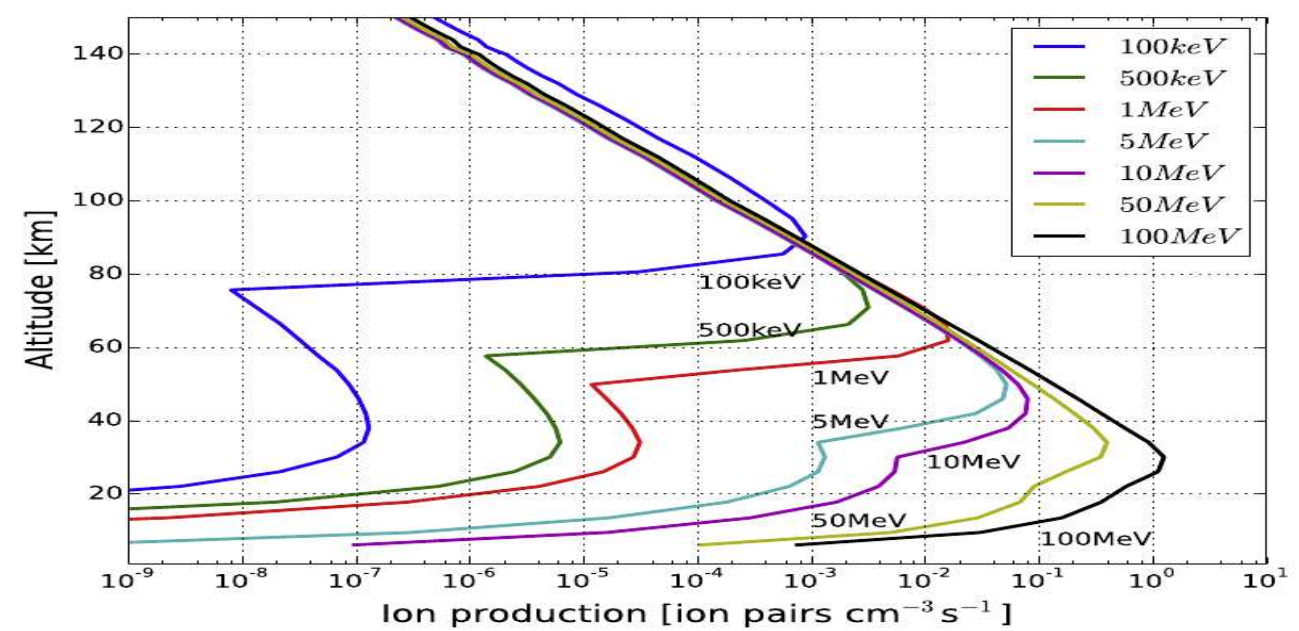

Figure 1: Ionization yields vs. altitude due to isotropic incidence of monoenergetic electrons in the energy range $100 \mathrm{keV}-100 \mathrm{MeV}$, as denoted in the legend.

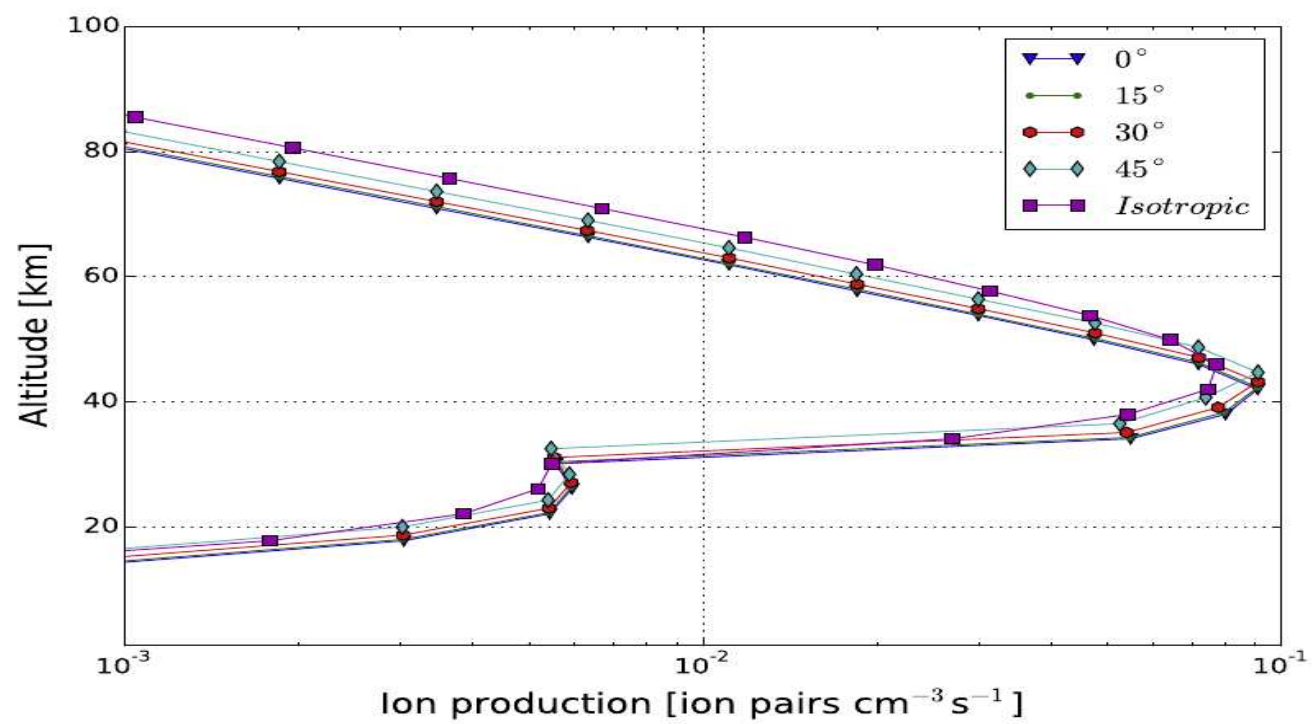

Figure 2: Ionization yelds vs. altitude $[\mathrm{km}]$ of electron with $10 \mathrm{MeV}$ energy for isotropic and various angles of incidence as denoted in the legend.

\section{Computation of ionization induced by EPs with arbitrary incidence}

On the basis of a quasi-analytical approach, based on re-computation of vertically derived ionization yields, we can compute the ionization yields for events with arbitrary incidence, details are given elsewhere [20]. The ionization yields $Y_{\alpha}\left(x^{\prime}, K\right)$ for a monoenergetic electrons with energy $K$ and with angle of incidence $\alpha$ is calculated:

$$
Y_{\alpha}(x, K)=Y\left(x^{\prime}, K\right) / \cos \alpha,
$$

where $x^{\prime}$ is the rescaled atmospheric depth, calculated as $x^{\prime}=x / \cos \alpha$ and $Y(x, K)$ is the ionization 
yields for electrons with vertical incidence computed with the CRAC:EPII model at depth $x$, details given elsewhere[21,22]. Let the intensity of electrons is $J(K, \alpha, \phi)=J_{0} f(\alpha, \phi)$ where $\alpha$ and $\phi$ are the the zenith and azimuth angles of incident electrons and $J_{0}$ is unit flux and the angular distribution is normalized to 1 i.e. $\int_{0}^{2 \pi} \int_{-\pi / 2}^{\pi / 2} \sin \alpha d \alpha d \phi=1$. The flux of electrons within a solid angle $d \Omega=\sin \alpha d \alpha d \phi$ is

$$
d F(K)=J_{0}(K) f(\alpha, \phi) \sin \alpha \cos \alpha d \alpha d \phi
$$

Accordingly the total flux of electrons is $F(K)=A \cdot J_{0}(K)$, where $A=\int_{0}^{2 \pi} \int_{0}^{1} f(\alpha, \phi) \cos \alpha d \cos \alpha d \phi$. Hence the ion production rate at depth $x$ is:

$$
I(x, K)=\rho(h) \int_{0}^{2 \pi} \int_{0}^{1} J_{0}(K) Y_{\alpha}(x, K) f(\alpha, \phi) \cos \alpha d \cos \alpha d \phi
$$

The corresponding ionization yield function $Y_{f}(x, K)$ for particles with arbitrary angular distribution $f(\alpha, \phi)$ in a way that $I(x, K)=Y_{f}(x, K) F(K) \rho(h)$, leads to:

$$
Y_{f}(x, K)=\frac{1}{A} \int_{0}^{2 \pi} \int_{0}^{1} Y_{\alpha}(x, K) f(\alpha, \phi) \cos \alpha d \cos \alpha d \phi
$$

In the case of axial symmetry $f(\alpha, \phi)$ is a function only on the zenith angle $\alpha$. Therefore:

$$
Y_{f}(x, K)=\frac{\int_{0}^{1} Y_{v}\left(x^{\prime}, K\right) d \cos \alpha}{\int_{0}^{1} f(\alpha) \cos \alpha d \cos \alpha}
$$

where $x^{\prime}$ is the rescaled atmospheric depth, calculated as $x^{\prime}=x / \cos \alpha$ and $Y_{v}$ is the ionization yield function for electrons with vertical incidence. It is easy to see that:

$$
f(\alpha)=\frac{n+1}{4 \pi} \cos ^{n} \alpha
$$

which in case of isotropic incidence (distribution) $n=0$, hence $f=1 / \pi$ leads to:

$$
Y_{i s o}(x, K)=2 \int_{0}^{1} Y_{v}\left(x^{\prime}, K\right) d \cos \alpha
$$

A good agreement between Monte Carlo simulations and the quasi-analytical approach is achieved (Fig.3)

The yield function $Y(x, K)$ is the response of the atmosphere, the ionization yields, to the mono-energetic unit flux of primary particles entering the Earth's atmosphere. One can see the essential contribution of Bremsstrahlung in the ionization yields, specifically at depths below 1 $\mathrm{g} / \mathrm{cm}^{2}$, which is explicitly considered in the yield function (Fig.4) The shapes of the ionization yield functions as a function of the altitude are similar to each other at depths greater than $5 \mathrm{~g} / \mathrm{cm}^{2}$, but different in the region of the upper atmosphere, where spikes exist due to large fluctuations of the computed energy deposit and the lack of secondary particles.

The differential ionization function $F$ (the integrand of Eq. 2.2, but $\rho(h)$ term), defined as a product of the ionization yield function (Fig.4) and a given spectrum of primary electrons is shown in Figure 5 for several atmospheric depths. Here, we consider a hard electron spectrum from [23], the details of computation are given elsewhere [21]. The differential ionization function $F$ allows 

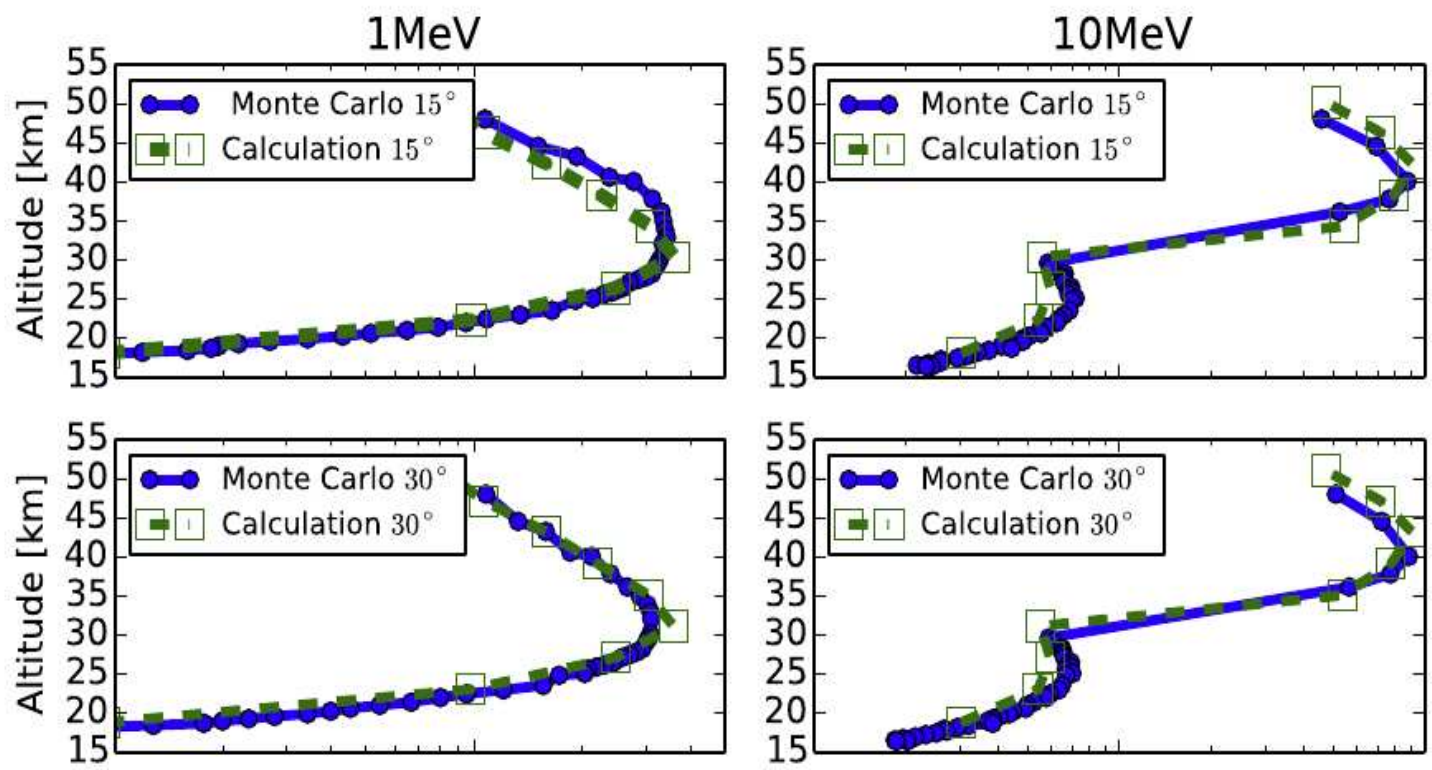

Figure 3: Comparison of ionization rates due to electrons with various angle of incidence and energy as denoted in the legend, computed with quasi-analytical approach Eq. (4) and PLANETOCOSMICS. The left hand panels denote electrons with energy $1 \mathrm{MeV}$, while right hand denote electrons with energy $10 \mathrm{MeV}$.

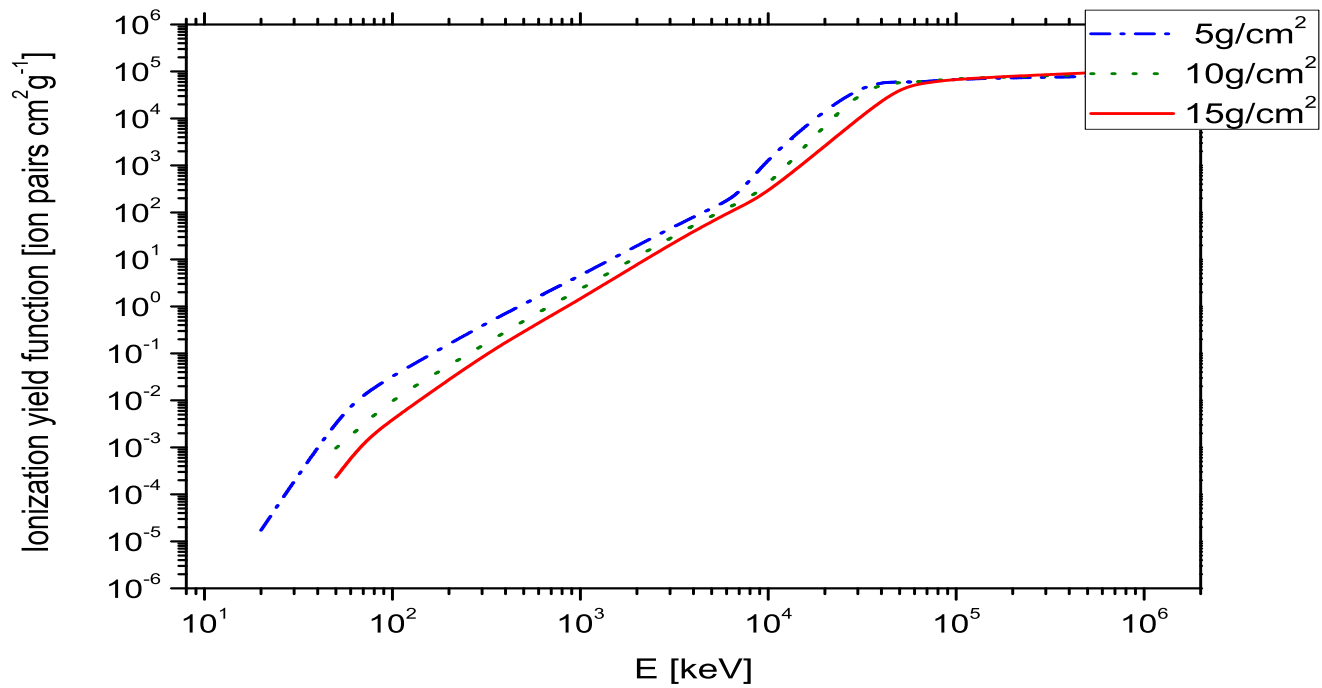

Figure 4: Ionization yield function for precipitating electrons with vertical incidence at several depths as denoted in the legend. The curves are smoothed over the computed data points.

one to estimate the most effective energy of primaries to induce ionization, which strongly depends on the atmospheric depth. Here, the integration is over the energy above $20 \mathrm{keV}$. One can see that the most effective energy of precipitating electrons to induce ionization strongly depends on the 


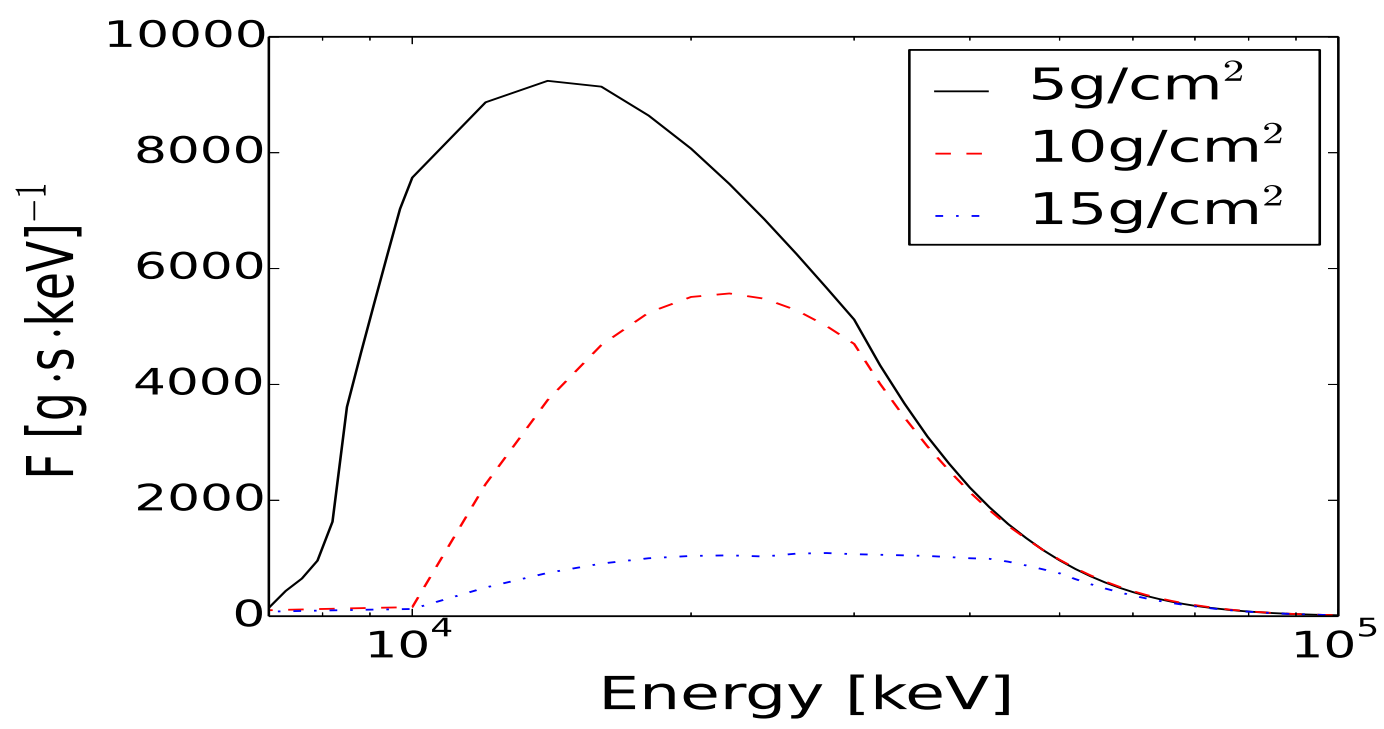

Figure 5: Differential ionization function $F$ for precipitating electrons at several depths as denoted in the legend.

atmospheric depth. The ionization at depth of about $5 \mathrm{~g} / \mathrm{cm}^{2}$ is dominated by electrons with energy of about $10 \mathrm{MeV}$, accordingly at depth of about $10 \mathrm{~g} / \mathrm{cm}^{2}$ is governed by particles with energy of about $200 \mathrm{MeV}$. The maximum shifts to higher energies with decreasing the altitude (increasing the depth). At depths of about $15 \mathrm{~g} / \mathrm{cm}^{2}$ the differential ionization function $F$ flattens, because of the diminishing number of high energy precipitating electrons.

\section{Conclusion}

Here, we have presented an upgraded full numerical model CRAC:EPII, which allows one to compute the ion production in the Earth's atmosphere due to relativistic and high energy precipitating electrons. The model is based on a full Monte Carlo simulations of propagation and interaction of precipitating electrons with the air using the PLANETOCOSMICS code. The model allows one to perform computations of ion production in the whole atmosphere, specifically in the stratosphere over the Globe. In fact, the model is an extension of cosmic ray ionization model CRAC. A convenient quasi-analytical approach for computation of the ionization yields for particles with arbitrary incidence, based on re-computation of vertically derived ionization yields is presented. A good agreement of the approach with direct simulations is achieved.

\section{Acknowledgements}

This work was supported by the Academy of Finland (project 272157, Center of Excellence ReSoLVE). 


\section{References}

[1] G. A. Bazilevskaya, I. G. Usoskin, E. Flückiger, R. Harrison, L. Desorgher, B. Bütikofer, M. Krainev, V. Makhmutov, Y. Stozhkov, A. Svirzhevskaya, N. Svirzhevsky and G. Kovaltsov, Cosmic ray induced ion production in the atmosphere, Space Science Reviews 137 (2008) 149-173.

[2] I. Mironova, K. Aplin, F. Arnold, G. Bazilevskaya, R. Harrison, A. Krivolutsky, K. Nicoll, E. Rozanov, E. Turunen and I. Usoskin, Energetic particle influence on the Earth's atmosphere, Space Science Reviews (2015) 96.

[3] P. T. Newell, T. Sotirelis and S. Wing, Seasonal variations in diffuse, monoenergetic, and broadband aurora, Journal of Geophysical Research: Space Physics 115 (2010), no. A3 A03216.

[4] G. Remenets and A. Astafiev, Solution uniquity of an inverse VLF problem: A case-study of the polar, ground-based, VLF radio signal disturbances caused by the ultra-energetic relativistic electron precipitations and of their southern boundaries, Advances in Space Research 58 (2016), no. 6 878-889.

[5] Y. Shprits, A. Drozdov, M. Spasojevic, A. Kellerman, M. Usanova, M. Engebretson, O. Agapitov, I. Zhelavskaya, T. Raita, H. Spence, D. Baker, H. Zhu and N. Aseev, Wave-induced loss of ultra-relativistic electrons in the van Allen radiation belts, Nature Communications 7 (2016) 12883.

[6] M. Rees, Auroral ionization and excitation by incident energetic electrons, Planetary and Space Science 11 (1963), no. 10 1209-1218.

[7] V. Makhmutov, G. Bazilevskaya and M. Krainev, Characteristics of energetic electron precipitation into the earth's polar atmosphere and geomagnetic conditions, Advances in Space Research 31 (2003), no. 4 1087-1092.

[8] R. Millan and R. Thorne, Review of radiation belt relativistic electron losses, Journal of Atmospheric and Solar-Terrestrial Physics 69 (2007), no. 3 362-377.

[9] R. Horne, M. Lam and J. Green, Energetic electron precipitation from the outer radiation belt during geomagnetic storms, Geophysical Research Letters 36 (2009), no. 19 L19104.

[10] B. Tsurutani, G. Lakhina and O. Verkhoglyadova, Energetic electron (>10 keV) microburst precipitation, $\sim 5-15$ s $x$-ray pulsations, chorus, and wave-particle interactions: A review, Journal of Geophysical Research: Space Physics 118 (2013), no. 5 2296-2312.

[11] Y. Miyoshi, S. Oyama, S. Saito, S. Kurita, H. Fujiwara, R. Kataoka, Y. Ebihara, C. Kletzing, G. Reeves, O. Santolik, M. Clilverd, C. Rodger, E. Turunen and F. Tsuchiya, Energetic electron precipitation associated with pulsating aurora: EISCAT and van Allen probe observations, Journal of Geophysical Research A: Space Physics 120 (2015), no. 4 2754-2766.

[12] L. Callis, Precipitating relativistic electrons: their long-term effect on stratospheric odd nitrogen levels, Journal of Geophysical Research 96 (1991), no. D2 2939-2976.

[13] E. Rozanov, L. Callis, M. Schlesinger, F. Yang, N. Andronova and V. Zubov, Atmospheric response to NOy source due to energetic electron precipitation, Geophysical Research Letters 32 (2005), no. 14 1-4.

[14] E. Turunen, P. Verronen, A. Seppälä, C. Rodger, M. Clilverd, J. Tamminen, C.-F. Enell and T. Ulich, Impact of different energies of precipitating particles on NOx generation in the middle and upper atmosphere during geomagnetic storms, Journal of Atmospheric and Solar-Terrestrial Physics 71 (2009), no. 10-11 1176-1189. 
[15] P. Verronen, C. Rodger, M. Clilverd and S. Wang, First evidence of mesospheric hydroxyl response to electron precipitation from the radiation belts, Journal of Geophysical Research: Atmospheres 116 (2011), no. 7 D07307.

[16] L. Desorgher, E. Flückiger, M. Gurtner, M. Moser and R. Bütikofer, A GEANT 4 code for computing the interaction of cosmic rays with the Earth's atmosphere, Internationl Journal of Modern Physics A 20 (2005), no. A11 6802-6804.

[17] J. Picone, A. Hedin, D. Drob and A. Aikin, NRLMSISE-00 empirical model of the atmosphere: Statistical comparisons and scientific issues, Journal of Geophysical Research: Space Physics $\mathbf{1 0 7}$ (2002), no. A12 1468.

[18] I. Usoskin and G. Kovaltsov, Cosmic ray induced ionization in the atmosphere: Full modeling and practical applications, Journal of Geophysical Research 111 (2006), no. D21206.

[19] H. Porter, C. Jackman and A. Green, Efficiencies for production of atomic nitrogen and oxygen by relativistic proton impact in air, The Journal of Chemical Physics 65 (1976), no. 1 154-167.

[20] A. Artamonov, I. Mironova, G. Kovaltsov, A. Mishev, E. Plotnikov and N. Konstantinova, Calculation of atmospheric ionization induced by electrons with non-vertical precipitation: Updated model crac-epii, Advances in Space Research 59 (2017), no. 9 2295-2300.

[21] A. Artamonov, A. Mishev and I. Usoskin, Model CRAC:EPII for atmospheric ionization due to precipitating electrons: Yield function and applications, Journal of Geophysical Research A: Space Physics 121 (2016), no. 2 1736-1743.

[22] A. Artamonov, A. Mishev and I. Usoskin, Atmospheric ionization induced by precipitating electrons: Comparison of new Monte Carlo model with parametrization driven model, Journal of Atmospheric and Solar-Terrestrial Physics 149 (2016) 161-166.

[23] V. Makhmutov, G. Bazilevskaya, Y. Stozhkov, A. Svirzhevskaya and N. Svirzhevsky, Catalogue of electron precipitation events as observed in the long-duration cosmic ray balloon experiment, Journal of Atmospheric and Solar-Terrestrial Physics 149 (2016) 258-276. 